\title{
Frecuencia de maloclusión en las clínicas odontopediátricas de la Universidad de Antioquia, Colombia, y de la Universidad Autónoma de San Luis Potosí, México
}

\author{
Gabriel Espinal Botero*, Esp. ${ }_{1}$, Ana Muñoz B., Est. Odont. ${ }_{2}$, Lizet Flores \\ M., Est. Odont., Margarita Ponce P., MsC. ${ }_{2}$, Jaime Nava C., MsC., Jaime \\ González Q., Esp.
}

${ }_{1}$ Facultad de Odontología, Universidad de Antioquia, Medellín, Colombia ${ }_{2}$ Facultad de Odontología, Universidad Autónoma de San Luis Potosí, San Luis Potosí, México

Recibido: 16 de septiembre del 2015 Aprobado: 21 de octubre del 2015

*Autor de correspondencia: Gabriel Espinal Botero. Universidad de Antioquia, Medellín. Calle 64 n. ${ }^{\circ}$ 52-59. Teléfono: (+57) 2196772. Correo electrónico: gabriel.espinal@udea.edu.co

Cómo citar este artículo: Espinal-Botero G, Muñoz A, Flores L, Ponce M, Nava J, González J. Frecuencia de maloclusión en las clínicas odontopediátricas de la Universidad de Antioquia, Colombia, y de la Universidad Autónoma de San Luis Potosí, México. Rev Nac Odontol. 2016:12(22):61-68. doi: http://dx.doi.org/10.16925/od.v12i22.1206

Resumen. Introducción: las maloclusiones son un conjunto de alteraciones en las que los controles anatomo-fisiológicos del sistema estomatognático se encuentran en desarmonía con los segmentos dentarios. Estudios epidemiológicos han mostrado que la maloclusión se presenta con tasas de prevalencia altas, de más del $60 \%$ de las poblaciones estudiadas. Las investigaciones sobre maloclusiones en poblaciones de 4 a 5 años reportan prevalencias del 70-80\%, que evolucionan hasta un $96,4 \%$ en adolescentes. Dacosta y Onyeaso reportan que, en el mundo la prevalencia de maloclusiones dentales, va desde del $65 \%$ hasta el $89 \%$. Objetivo: comparar el tipo de maloclusión que se presenta con más frecuencia en las clínicas de odontopediatría de la Universidad Autónoma de San Luis Potosí y de la Universidad de Antioquia. Metodología: se llevó a cabo un estudio observacional de tipo transversal y comparativo con un muestreo por conveniencia; se examinaron 200 pacientes por Facultad, con edades entre 4 y 15 años. Resultados: se observó mayor frecuencia de clase I tipo 1 en el grupo 1, con un porcentaje del 52,7\%, mientras que, en el grupo 2, las maloclusiones predominantes fueron la clase II div.1, con un porcentaje del $23,6 \%$, y la clase iII con 22,5\%. Conclusiones: los resultados de cada población fueron diferentes. Se recomienda que el diagnóstico de las alteraciones oclusales en la población pediátrica se realice de manera oportuna, permitiendo implementar programas de atención que incluyan terapias como la ortopedia funcional de los maxilares, para una rehabilitación temprana del sistema estomatognático.

Palabras clave: apiñamiento posterior, erupción dentaria, impactación, prevalencia, segundo molar inferior. 


\title{
Malocclusion Frequency in Odontopediatric Clinics of the Universidad de Antioquia, Colombia, and the Universidad Autónoma de San Luis Potosí, Mexico
}

\begin{abstract}
Introduction: malocclusions are a series of disorders in which the anatomic physiologic controls of the stomatognathic system are in disharmony with the dental segments. Epidemiological studies show that malocclusion have high prevalence rates of over $60 \%$ in the studied populations. The malocclusion research in populations of 4 to 5 years old report prevalence of 70 to $80 \%$, which evolve to $96,4 \%$ in teenagers. Dacosta and Onyeaso report that the prevalence of dental malocclusions worldwide ranges from 65 to $89 \%$. Objective: compare the type of malocclusion that is most frequently presented in odontopediatric clinics of the Autonomous University of San Luis Potosi and the University of Antioquia. Methodology: an observational study was conducted of transversal and comparative type with a convenience sample; a higher frequency of class I type 1 was seen in group 1, with a percentage of 52,7\%, while in group 2, the predominant malocclusions where class II div. 1, with a percentage of $23,6 \%$ and class III with $22,5 \%$. Conclusion: the results in each population were different. The diagnosis of occlusal alterations is recommended to be performed in a timely manner to allow the implementation of assistance programs that include therapy and functional orthopedics of the jawbone for an early rehabilitation of the stomatognathic system.
\end{abstract}

Keywords: posterior crowding, dental eruption, impact, prevalence, second inferior molar.

\section{Frequência de maloclusão nas clínicas odontopediátricas da Universidade de Antioquia, Colombia, e da Universidade Autónoma de San Luis Potosí, México}

Resumo. Introdução: as maloclusões são um conjunto de alterações nas quais os controles anatomo-fisiológicos do sistema estomatognático estão em desarmonia com os segmentos dentários. Estudos epidemiológicos têm mostrado que a maloclusão se apresenta com taxas de prevalência altas, mais de $60 \%$ das populações estudadas. As pesquisas sobre maloclusões em populações de 4 a 5 anos relatam prevalências do $70-80 \%$, que evoluem até $96,4 \%$ em adolescentes. Dacosta e Onyeaso relatam que no mundo a prevalência de maloclusões dentais, vai desde $65 \%$ até $89 \%$. Objetivo: comparar o tipo de maloclusão apresentada com maior frequência nas clínicas de odontopediatria da Universidade Autónoma de San Luis Potosí e da Universidade de Antioquia. Metodologia: foi feito um estudo observacional de tipo transversal e comparativo com uma amostragem por conveniência; examinaram-se 200 pacientes por Faculdade, na faixa etária de 4 e 15 anos. Resultados: observou-se maior frequência de classe I tipo 1 no grupo 1, com uma percentagem de 52,7\%, enquanto que, no grupo 2, as maloclusões predominantes foram a classe II div.1, com uma percentagem de $23,6 \%$, e a classe III com 22,5\%. Conclusões: os resultados de cada população foram diferentes. Recomenda-se que o diagnóstico das alterações oclusais na população pediátrica seja feita oportunamente, permitindo implementar programas de atendimento que incluam terapias como a ortopedia funcional dos maxilares, para uma reabilitação precoce do sistema estomatognático.

Palavras-chave: apinhamento posterior, erupção dentária, impactacão, prevalência, segundo molar inferior. 


\section{Introducción}

Los estándares de belleza varían según la etapa de vida, sin embargo, la estética siempre ha sido motivo de preocupación de los individuos. Para el ser humano, la comunicación y la sonrisa son la puerta de entrada a las relaciones humanas, la autoestima y la calidad de vida, de ahí que el cuidado de la boca y la posición dentaria cobran especial importancia [1].

La palabra oclusión se refiere al contacto entre dientes de arcadas opuestas al cerrarse los maxilares (oclusión estática) y durante los diferentes movimientos mandibulares (oclusión dinámica). La localización de los contactos entre dientes opuestos (contactos oclusales) varía como resultado tanto de las diferencias en el tamaño y la forma de los dientes y los maxilares, como de la posición de los dientes en los maxilares. Una oclusión funcional es un estado en el cual las superficies oclusales no presentan obstáculos o interferencias para los movimientos mandibulares, y en el que exista la máxima interdigitación cuspídea en oclusión céntrica, respetando todas las reglas de la fisiología, anatomía y neurofisiología humanas $[2,3]$.

Dentro de las alteraciones bucales de mayor prevalencia, se encuentran las maloclusiones, que afectan a un amplio sector de la población, por lo que son consideradas un problema de salud pública; sin embargo, su importancia se establece no solo por el número de personas que la presentan, sino, además, por los efectos nocivos que pueden generar en el aparato estomatognático, tales como ruido articular, dolor muscular y trastornos del habla $[4,5,6]$.

Una maloclusión se identifica cuando los controles anatomo-fisiológicos del sistema estomatognático se encuentran en desarmonía con los segmentos dentarios, pudiendo estar presentes desde el origen del individuo, o bien instalándose durante las primeras etapas de vida, cobrando magnitudes diferentes con el paso del tiempo [7].

Por lo general, la palabra normal se usa para expresar un patrón de referencia o situación óptima en las relaciones oclusales y, aunque no es lo que encontramos con mayor frecuencia en nuestros pacientes, sí se considera el patrón más adecuado para cumplir la función masticatoria y preservar la integridad de la dentición a lo largo de la vida, en armonía con el sistema estomatognático [1].

Las maloclusiones se encuentran determinadas por múltiples factores, en especial de índole hereditaria, como son las diferencias maxilomandibulares del crecimiento y las malposiciones dentarias en cada arcada. En estas influyen, entre otros factores, las alteraciones en la cronología de la erupción dentaria, la pérdida prematura de dientes y la caries dental, originando ocasionalmente que los órganos dentarios no cumplan ciertos parámetros considerados "normales" en la oclusión [8]. Los hábitos orales como la succión digital y el hábito de empuje lingual y labial, entre otros, se consideran condicionantes en el desarrollo de la maloclusión; su modificación o eliminación posibilitan una mejoría en el desarrollo de la oclusión [9].

Estudios epidemiológicos han mostrado que la maloclusión se presenta con tasas de prevalencia considerablemente altas: más del $60 \%$ de la población la desarrolla [10,11]. Las investigaciones sobre maloclusiones en niños de 4 a 5 años reportan prevalencias del $70-80 \%$ [12], llegando a un $96,4 \%$ en adolescentes. Murrieta, Dacosta y Onyeaso [4, 13, 14] reportan que, en el mundo, la prevalencia de maloclusiones dentales es de alta frecuencia: desde el $65 \%$ hasta el $89 \%$ de la población se ve afectada por ella.

Murrieta [9] afirma que la clase I con apiñamiento es la más frecuente entre adolescentes mexicanos, con una participación del 72,8\%; se estima que dicha prevalencia a nivel poblacional oscila entre el $69,5 \%$ y el $76,2 \%$. La clase II es la segunda más frecuente, con un $13,5 \%$ de los casos detectados, y finalmente la clase III con un $10,1 \%$; cifras que indican que la frecuencia de maloclusiones clase I con apiñamiento es cinco a siete veces mayor en comparación con los casos de maloclusión clase II y III, respectivamente [4]. Otros autores reportan que el 37\% de los niños de 12 años presentan sobremordida horizontal mayor de $3 \mathrm{~mm}$, y el $4,6 \%$ de los adolescentes entre 12 y 15 años presenta una sobremordida mayor de $6 \mathrm{~mm}$ [7].

En cuanto a la población colombiana, según el IV Estudio Nacional de Salud Bucal (ENSAB IV), realizado en el 2014 [15], se encontró que, en la población de 5 años, la relación molar de plano terminal recto corresponde a más del $70 \%$, y el $21 \%$ corresponde a escalón mesial bilateral; en esta misma población, el 71\% de los niños presentaba una sobremordida horizontal normal, el 12,5\% aumentada, y el 3,7\% cruzada anterior, y además, cerca del 3,9\% presentaba mordida cruzada posterior bilateral. En el grupo de adolescentes de 12 a 15 años de todas las condiciones de la oclusión estudiadas, la de mayor 
prevalencia es el apiñamiento en ambos arcos, con presencia del $56,1 \%$ a los 12 años y del $68 \%$ a los 15 años; además, en la sobremordida horizontal, la relación de borde a borde a los 12 años es del 3,1\% y, a los 15 años, del 5,2\%; la mordida cruzada anterior es del $3,7 \%$ a los 12 años y del $6,47 \%$ a los 15 años. En otro estudio realizado en Medellín, en pacientes que asisten al Colegio Universidad Cooperativa de Colombia, los resultados indican que la maloclusión con mayor prevalencia es la clase II división 1 (43\%), seguida de la clase I (35\%), clase II división 2 (13\%) y en menor porcentaje la clase III (9\%) [16].

La dentición temporal ha demostrado su importancia para el futuro desarrollo de la dentición permanente, por lo que es necesario vigilar su crecimiento y desarrollo y la influencia sobre esta, de factores de riesgo que van modificando notablemente la morfología cráneofacial, que provoca - desde edades tempranas - relaciones oclusales inadecuadas. La experiencia ha demostrado que el tratamiento de las maloclusiones en edades tempranas es eficiente y con resultados más estables que en edades más avanzadas, y es por eso que el esfuerzo principal como profesionales debe estar encaminado a determinar en dichas edades los factores que las propicien, de tal manera que se reduzcan mediante un adecuado y oportuno diagnóstico, además del incremento de las acciones preventivas-interceptivas, fundamentalmente en la población infantil [17].

El propósito del presente estudio es comparar la prevalencia de maloclusión en la población infantil que consultó a las clínicas de odontopediatría de la Universidad de Antioquia y de la Universidad Autónoma de San Luis Potosí, con el propósito de elaborar indicadores para el establecimiento de métodos preventivos e interceptivos dirigidos a la población infantil.

\section{Métodos}

Se llevó a cabo un estudio observacional de tipo transversal y comparativo con un muestreo por conveniencia, utilizando análisis de frecuencias y porcentajes para analizar los resultados. Para ello, se analizó la oclusión de una primera muestra de
200 niños que asistieron a consulta a la Clínica de Estomatología Pediátrica de pregrado de la Facultad de Estomatología de la Universidad Autónoma de San Luis Potosí (grupo 1). De igual manera, se analizó la oclusión de la segunda muestra de 200 pacientes en la Clínica del Niño y el Adolescente de la Facultad de Odontología de la Universidad de Antioquia (grupo 2). Para realizar el estudio, primero se solicitó a los padres la lectura del consentimiento informado y su autorización, además del asentimiento dirigido a los niños. Los criterios de inclusión para la muestra fueron pacientes que tuviesen su oclusión establecida, en un rango de edad de 4 a 15 años, y los criterios de exclusión fueron: pacientes que presentaban algún tipo de síndrome o malformaciones cráneofaciales; primeros molares permanentes y segundos molares temporales perdidos o destruidos o con mantenedores de espacio con banda o corona, que impidiesen observar la relación molar; dientes anteriores temporales con caries de la infancia temprana o perdidos, y niños con prótesis en anteriores, zapatillas distales y tratamientos de ortodoncia previos.

El examen bucal de las dos muestras lo realizaron los mismos examinadores, quienes fueron previamente entrenados e instruidos por un experto en el área de cada facultad para asegurar la confiabilidad de la información. Se revisó a cada uno de los pacientes en una unidad dental, completamente reclinados, con iluminación directa, y se utilizó visión directa además de espejo y explorador. Se les pidió abrir la boca y cerrarla con la lengua apoyada en la parte más posterior del paladar. La recolección de datos se hizo en un formato de ficha clínica impresa, en la cual, además de anotar el tipo de maloclusión que presentaba cada paciente, se diligenció nombre completo, edad y sexo. Los criterios a observar (que se anotaron en la ficha clínica) en dentición temporal se presentan en la tabla 1 [18]; para evaluar el tipo de oclusión en dentición mixta o permanente se aplicaron dos sistemas de clasificación: Anderson (tabla 2) [19] y Angle [20] (tabla 3). En cuanto a este último, se agregó la clasificación "Maloclusión clase I tipo 0" para describir los arcos que presentaran los dientes anteriores bien alineados. 
Tabla 1. Parámetros para clasificar la dentición temporal

\begin{tabular}{|l|l|}
\hline \multicolumn{1}{|c|}{ Condición } & \multicolumn{1}{c|}{ Descripción } \\
\hline Arco de Baume & Abierto/cerrado \\
\hline Línea media & Normal/desviada \\
\hline Relación canina & Clase I/clase II/clase III \\
\hline Espacios de primate & Superior/inferior \\
\hline Plano terminal & Vertical/mesial/distal/mesial exagerado \\
\hline Relación incisiva & $\begin{array}{l}\text { Normal/sobremordida horizontal/sobre- } \\
\text { mordida vertical/mordida borde a borde }\end{array}$ \\
\hline $\begin{array}{l}\text { Relación transversal } \\
\text { posterior }\end{array}$ & $\begin{array}{l}\text { Normal/cruzada unilateral/cruzada uniden- } \\
\text { tal/cruzada multidental/cruzada bilateral }\end{array}$ \\
\hline
\end{tabular}

Fuente: [18]

Tabla 2. Clasificación de Anderson de acuerdo con el tipo de oclusión dental

\begin{tabular}{|l|l|}
\hline Maloclusión clase $\mathbf{I}$ & \multicolumn{1}{|c|}{ Descripción } \\
\hline Tipo 0 & Dientes anteriores bien alineados. \\
\hline Tipo 1 & Dientes anteriores apiñados y rotados. \\
\hline Tipo 2 & $\begin{array}{l}\text { Dientes anterosuperiores protruídos y } \\
\text { espaciados. }\end{array}$ \\
\hline Tipo 3 & Dientes anteriores en mordida cruzada. \\
\hline Tipo 4 & Dientes posteriores en mordida cruzada. \\
\hline Tipo 5 & $\begin{array}{l}\text { Pérdida de espacio en los segmentos } \\
\text { posteriores. }\end{array}$ \\
\hline
\end{tabular}

Fuente: [19]

Tabla 3. Clasificación de acuerdo con las características de la maloclusión clase I de Angle

\begin{tabular}{|l|l|}
\hline \multicolumn{1}{|c|}{ Condición } & \multicolumn{1}{c|}{ Descripción } \\
\hline $\begin{array}{l}\text { Clase I } \\
\text { (normoclusión) }\end{array}$ & $\begin{array}{l}\text { Cúspide mesiovestibular del primer molar } \\
\text { permanente superior ocluye en el surco mesio- } \\
\text { vestibular del primer molar inferior. }\end{array}$ \\
\hline $\begin{array}{l}\text { Clase II } \\
\text { (distoclusión) }\end{array}$ & $\begin{array}{l}\text { Cúspide mesiovestibular del primer molar per- } \\
\text { manente superior ocluye en la vertiente distal } \\
\text { del segundo premolar inferior. }\end{array}$ \\
\hline $\begin{array}{l}\text { Clase III } \\
\text { (mesioclusión) }\end{array}$ & $\begin{array}{l}\text { Se presenta cuando en oclusión habitual el } \\
\text { primer molar inferior permanente se encuentra } \\
\text { en sentido mesial en relación con el primer } \\
\text { molar permanente superior. }\end{array}$ \\
\hline
\end{tabular}

Fuente: [20]

\section{Resultados}

En la tabla 4 y las figuras 1 y 2 , se muestran los resultados del tipo de maloclusión más prevalente en ambas poblaciones, después del análisis de frecuencias y porcentajes.

En el grupo 1, el total de niños con dentición mixta o permanente revisados fue de 167 (56,2\% del sexo femenino), y en el grupo 2 se examinaron
191 niños (54,4\% del sexo femenino). La frecuencia por tipo de maloclusión identificada en los dos grupos obtuvo los porcentajes que se muestran en la tabla 4.

Tabla 4. Comparación porcentual de maloclusiones entre población del grupo 1 y de grupo 2

\begin{tabular}{|l|c|c|}
\hline $\begin{array}{c}\text { Clasificación de la } \\
\text { maloclusión }\end{array}$ & $\begin{array}{c}\text { Grupo 1 } \\
\text { (\%) }\end{array}$ & $\begin{array}{c}\text { Grupo 2 } \\
\text { (\%) }\end{array}$ \\
\hline Clase I tipo 0 & 1,8 & 0,5 \\
\hline Clase I tipo 1 & 52,7 & 18,8 \\
\hline Clase I tipo 2 & 15,0 & 16,8 \\
\hline Clase I tipo 3 & 4,8 & 5,2 \\
\hline Clase I tipo 4 & 3,6 & 3,7 \\
\hline Clase I tipo 5 & 0 & 0,5 \\
\hline Clase II div. 1 & 13,2 & 23,6 \\
\hline Clase II div. 2 & 3,6 & 8,4 \\
\hline Clase III & 5,4 & 22,5 \\
\hline
\end{tabular}

Fuente: elaboración propia

De acuerdo con los resultados de la tabla 4, en el grupo 1, la frecuencia más alta de maloclusión corresponde a la clase I tipo 1, seguido de la clase I tipo 2, y en tercer lugar, la clase II div. 1. En el grupo 2, la frecuencia más alta de maloclusión corresponde a la clase II div. 1 , seguida de la clase III, y en tercer lugar, la clase I tipo 1.

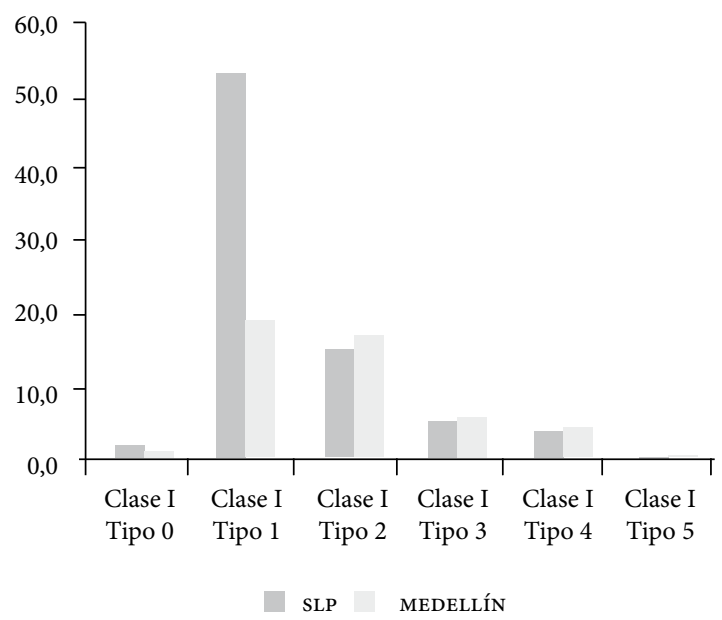

Figura 1. Comparación de la oclusión en dentición mixta o permanente: clase I, tipo 0 a 5. Población infantil de 6 a 14 años en el grupo 1 (SLP) y el grupo 2 (Medellín) Fuente: elaboración propia 


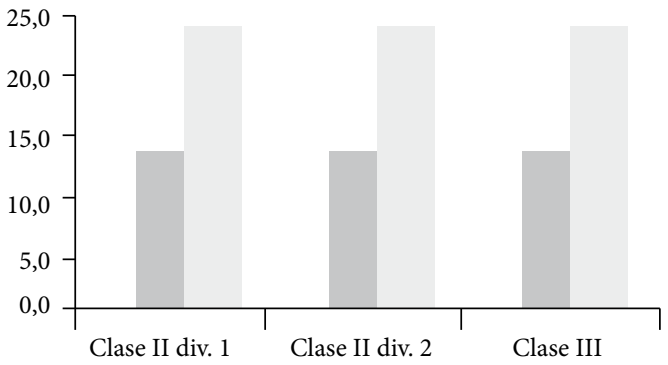

SLP MEDELLÍN

Figura 2. Comparación de la oclusión en dentición mixta o permanente: clase II div. 1-2 y clase III. Población infantil de 6 a 14 años en el grupo 1 (SLP) y el grupo 2 (Medellín)

Fuente: elaboración propia

En cuanto a la dentición temporal, en el grupo 1 el total de niños revisados fue de 33 (20 del sexo femenino, 60,6\%), y en el grupo 2 se examinaron 9 pacientes (7 del sexo femenino, 78\%).

En la tabla 5, se aprecian las diferencias existentes entre los arcos de acuerdo con la clasificación de Baume. El mayor porcentaje de arcos abiertos se encontró en el grupo 2, con $89 \%$, a diferencia de lo encontrado en el grupo 1, que presentó un $45 \%$ de arcos abiertos y un $55 \%$ de arcos cerrados. En esta tabla 5 , también se observa que el grupo 1 presentó una línea media normal en el $91 \%$ de los casos, mientras que en el grupo 2 fue del $67 \%$. En cuanto a la relación canina, la mayor frecuencia correspondió a la clase 1, presente en el 79\% de los casos del grupo 1 y en el 55\% del grupo 2 . El segundo lugar lo ocupó la clase 3 , con un $44 \%$ en el grupo 2 y un $15 \%$ en el grupo 1.

También se muestra la presencia o ausencia de los espacios de primate. Se observaron resultados parecidos en ambos grupos: la ausencia de espacios alcanzó el $67 \%$ en el grupo 2 y el $61 \%$ en el grupo 1. La presencia de espacios se encontró en el $39 \%$ de los casos del grupo 1 y en el 33\% del grupo 2 . De igual forma, se observa que la mayor frecuencia de plano terminal fue el mesial, presentándolo el $61 \%$ del grupo 1 y el 56\% del grupo 2. En segundo lugar, el plano terminal vertical, $30 \%$ en el grupo $1 \mathrm{y}$ $22 \%$ en el grupo 2 , aunque el plano terminal mesial exagerado también obtuvo $22 \%$ en el grupo 2 .

En la tabla 5, también podemos apreciar que el $61 \%$ del grupo 1 tiene relación incisiva normal, 24\% de relación incisiva vertical, $9 \%$ de relación horizontal y $6 \%$ de mordida borde a borde; mientras que, en el grupo 2, la frecuencia más alta correspondió a la relación incisiva horizontal, con $44 \%$, seguida de la relación incisiva normal con $33 \%$ y, por último, la mordida borde a borde con $22 \%$.

Así mismo se ilustran los porcentajes obtenidos de la relación transversal posterior. Los porcentajes más altos correspondieron a una relación transversal posterior normal, $94 \%$ en el grupo $1 \mathrm{y}$ $67 \%$ en el grupo 2; en este último, también se presentó $22 \%$ de mordida cruzada unilateral y $11 \%$ de mordida cruzada unidental.

Tabla 5. Comparación porcentual de los parámetros para clasificar la dentición temporal entre el grupo 1 y el grupo 2

\begin{tabular}{|c|c|c|c|}
\hline Parámetro & Clasificación & $\begin{array}{c}\text { Frecuencia } \\
\text { Grupo } 1 \\
(\%)\end{array}$ & $\begin{array}{c}\text { Frecuencia } \\
\text { Grupo } 2 \\
(\%) \\
\end{array}$ \\
\hline Arco de Baume & $\begin{array}{l}\text { Abierto } \\
\text { Cerrado } \\
\text { Apiñado }\end{array}$ & $\begin{array}{c}45 \\
55 \\
0 \\
\end{array}$ & $\begin{array}{c}89 \\
0 \\
11\end{array}$ \\
\hline Línea media & $\begin{array}{l}\text { Normal } \\
\text { Desviada } \\
\end{array}$ & $\begin{array}{c}91 \\
9 \\
\end{array}$ & $\begin{array}{l}67 \\
33 \\
\end{array}$ \\
\hline Relación canina & $\begin{array}{l}\text { Clase } 1 \\
\text { Clase } 2 \\
\text { Clase } 3 \\
\end{array}$ & $\begin{array}{c}79 \\
6 \\
15 \\
\end{array}$ & $\begin{array}{c}55 \\
1 \\
44 \\
\end{array}$ \\
\hline $\begin{array}{l}\text { Espacios de } \\
\text { primate }\end{array}$ & $\begin{array}{l}\text { Presentes } \\
\text { Ausentes }\end{array}$ & $\begin{array}{l}39 \\
61 \\
\end{array}$ & $\begin{array}{l}33 \\
67 \\
\end{array}$ \\
\hline Plano terminal & $\begin{array}{l}\text { Vertical } \\
\text { Distal } \\
\text { Mesial } \\
\text { Mesial exagerado }\end{array}$ & $\begin{array}{c}30 \\
3 \\
61 \\
6 \\
\end{array}$ & $\begin{array}{c}22 \\
0 \\
56 \\
22 \\
\end{array}$ \\
\hline $\begin{array}{l}\text { Relación } \\
\text { incisiva }\end{array}$ & $\begin{array}{l}\text { Normal } \\
\text { Vertical } \\
\text { Horizontal } \\
\text { Borde a borde }\end{array}$ & $\begin{array}{c}61 \\
24 \\
9 \\
6 \\
\end{array}$ & $\begin{array}{c}33 \\
0 \\
44 \\
22 \\
\end{array}$ \\
\hline $\begin{array}{l}\text { Relación trans- } \\
\text { versal posterior }\end{array}$ & $\begin{array}{l}\text { Normal } \\
\text { Cruzada unilateral } \\
\text { Cruzada unidental } \\
\text { Cruzada multi- } \\
\text { dental } \\
\text { Cruzada bilateral }\end{array}$ & $\begin{array}{c}94 \\
6 \\
0 \\
0 \\
0\end{array}$ & $\begin{array}{c}67 \\
22 \\
11 \\
0 \\
0\end{array}$ \\
\hline
\end{tabular}

Fuente: elaboración propia

\section{Discusión}

De acuerdo con los resultados, la maloclusión prevalente en el grupo 1 es la clase I tipo 1, que se presenta en el $53 \%$ de los niños con dentición mixta o permanente examinados, en lo que coincide Murrieta [9], quien afirma que la clase I con apiñamiento es la más frecuente, pues se presenta en el 72,8\% de los casos del grupo 1. Así mismo, este resultado concuerda con el estudio hecho por 
la UNAM que relacionó la clase dental con el sexo y encontró que, del $52,8 \%$ de los pacientes con clase I, el $35,3 \%$ son de sexo femenino. En clase II encontramos el 33,9\% de los pacientes, de los cuales el $21,5 \%$ son mujeres. En clase III la frecuencia fue de $13,3 \%$ de pacientes, con un $7,9 \%$ de sexo femenino [3].

Estos resultados son similares a los obtenidos en un estudio elaborado por la Universidad Central de Venezuela, en el que el $64,30 \%$ de los pacientes pediátricos, con edades comprendidas entre 5 y 12 años, presentó maloclusión clase I, 20,67\% clase II y $15,03 \%$ clase III. El tipo prevalente fue el tipo 1, en el $62,66 \%$ del total de pacientes con clase I [18].

Por su parte, en su investigación sobre maloclusiones en niños y adolescentes de caseríos y comunidades nativas de la Amazonía de Ucayali, Perú, Aliaga y Matta [19], encontraron resultados que concuerdan con los anteriores, en los cuales la más frecuente fue la maloclusión clase I $(59,6 \%)$, seguida por la maloclusión clase II $(18,5 \%)$, la oclusión normal $(14,5 \%)$ y la maloclusión clase III (7,5\%).

En el grupo 2, los porcentajes de maloclusión más altos correspondieron a la clase II div. 1 $(23,6 \%)$, seguida de la clase III $(22,5 \%)$ y la clase I tipo $1(18,8 \%)$. Este porcentaje coincide con los resultados del estudio realizado por Bermúdez [16], en el cual la clase II div. 1 se presentó en un $43 \%$ de los casos, seguida de la clase i con un $35 \%$, la clase II div. 2 con $13 \%$ y, en un menor porcentaje, la clase III con el $9 \%$.

Por otro lado, y en contraste con estos resultados, se encuentra el estudio realizado por la Universidad Cooperativa de Colombia, en la que Botero et al. [20] establecen que la maloclusión más prevalente es la clase i en el 55\% de los casos, seguida de la clase iI con el $32,5 \%$ y, en menor porcentaje, la clase III con 12,6\%. De igual forma, Urrego y Jiménez [21] mencionan en su estudio sobre la oclusión dental en 436 escolares de Envigado, Antioquia, cuyas edades comprendían entre los 5 y los 12 años de edad, que la maloclusión con mayor prevalencia para la dentición permanente fue la maloclusión clase I, con $49,7 \%$, la maloclusión clase II con $43,5 \%$ y la maloclusión clase III con $6,8 \%$.

\section{Conclusiones}

Las alteraciones oclusales surgen en edades tempranas y para su resolución dependen de un abordaje temprano, ya que en la mayoría de los casos pueden ser prevenibles a través del cuidado de la ejercitación normal de las funciones del aparato estomatognático. En nuestro estudio, en el caso con el resultado de mayor prevalencia en el grupo 1 , en relación con la clase I tipo 1 , se debe insistir - de acuerdo con la filosofía de la rehabilitación neurooclusal- en la eficiencia masticatoria como el estímulo a través de alimentos duros y fibrosos, sin descuidar las otras funciones y los factores que pueden ser el origen de la diversidad de maloclusiones resultantes.

En el grupo 2, ante la alta prevalencia de clase II div. 1 y clase III, sabemos que, en cuanto a la primera, las causas son en su mayoría hábitos, por eso es necesario vigilar que estos no se instalen o bien corregirlos oportunamente para que la alteración no cobre magnitudes severas. En la clase iII tanto el factor ambiental como la deglución atípica son las causas que deben ser cuidadas, pues el porcentaje de pacientes con este hábito es alto en la etapa del recambio dentario. Aunque se tenga antecedente genético, el abordaje temprano logra los objetivos terapéuticos con más plenitud.

De acuerdo con lo anterior, se debe vigilar el desarrollo dentario a la par del crecimiento, que se conduzca dentro de parámetros de normalidad, eliminando a tiempo las causas que pudieran afectarlo. Es función del médico estomatólogo y más específicamente del odontopediatra y del ortopedista funcional, ya que ambos actúan según principios predominantemente preventivos, que ambicionan sostener la salud, previniendo la enfermedad, aunque se trate de causas genéticas. Cuanto más temprano se aplique el tratamiento, más simples y plenos serán los resultados, reduciendo la necesidad en un futuro de tratamientos correctivos complejos y de elevado costo, a los que no toda la población tiene alcance.

\section{Referencias}

[1] Di Santi de Modano, Vázquez VB. Maloclusión clase I: definición, clasificación, características clínicas y tratamiento. Rev Latinoam Ortod y Odontoped. 
2003:1-24. Disponible en: https://www.ortodoncia. ws/publicaciones/2003/pdf/art8.pdf

[2] Díez Cubas C. Anatomía dental para higienistas de atención primaria. Madrid: Visión Net. [Internet]. 2010. [Citado 2015 sep 16] Disponible en: https://books.google.com.co/books?id=wQW5e0 KsXyoC\&pg=PP $1 \& \operatorname{lpg}=\mathrm{PP} 1 \& \mathrm{dq}=$ Anatom $\% \mathrm{C} 3 \%$ $\mathrm{ADa}+$ dental+para+higienistas+de+atenci\% $\mathrm{C} 3 \%$ $\mathrm{B} 3 \mathrm{n}+$ primaria\&source $=$ bl\&ots $=$ rkJanes NXO\& sig=i_epSTUFdFSp 4 ACWHhEUNiX5j14 \&hl=es $-419 \& \mathrm{sa}=\mathrm{X} \& \mathrm{ved}=0 \mathrm{ahUKEwiO66 \textrm {W }} 8$ ndXLAhVBVz4KHUJYBugQ6AEIGzAA\#v=one page $\& \mathrm{q}=$ Anatom $\%$ C $3 \% \mathrm{ADa} \% 20$ dental $\% 20$ para $\% 20$ higienistas $\% 20 \mathrm{de} \% 20$ atenci $\%$ C $3 \%$ $\mathrm{B} 3 \mathrm{n} \% 20$ primaria\&f=false

[3] Talley M, Katagiri M, Pérez HE. Casuística de maloclusiones clase I, clase II y clase III según Angle en el Departamento de Ortodoncia de la UnAM. Rev Odontol Mex. 2007;11(4):175-80.

[4] Murrieta J. et al. Prevalencia de maloclusiones dentales en un grupo de adolescentes mexicanos y su relación con la edad y el género. Fund Act Odontol Venez. 2007;45(1): 1-7. Disponible en http://www. actaodontologica.com/ediciones/2007/1/pdf/maloclusiones_dentales.pdf

[5] Díaz Morell JE et al. Maloclusiones, signos y síntomas de trastornos temporomandibulares en adolescentes de la Parroquia Jusepín. Monagas, Venezuela. Corr Cient Méd. 2012;16(4):1-14.

[6] Rodríguez Peña NV. Anomalías de la oclusión y trastornos en la articulación de la palabra. Rev Cub Ortod. 2000;15(2):86-93.

[7] Villalba E, Gutiérrez DH, Díaz R. Evaluación de la relación maxilomandibular, parte clave del diagnóstico ortodóncico. Rev Latinoam Ortod y Odontoped. 2009;23(4):432-8.

[8] Taboada O. Prevalencia de maloclusiones y trastornos del habla en una población preescolar del oriente de la Cd. de México. Bol Med Hosp Infant Mex. 2011;68(6):425-30.

[9] Murrieta JF. Características de la dentición primaria y su posible influencia en el desarrollo de la oclusión en niños de 3 a 5 años de edad. Rev Especializ Cien Sal. 1999;2(1):21-6.

[10] Cartes R, Araya E, Valdés C. Maloclusiones y su impacto psicosocial en estudiantes de un liceo intercultural. Int J Odontostomat. 2010;4:65-70.

[11] Martin CC, Barberia LE, González SA, Rioboó GR. Prevalencia de maloclusiones en niños de la Comunidad Autónoma de Madrid según el índice estético dental. Rev. Esp Ortod. 2009;39(2):91-102.

[12] Quirós O. Características de la oclusión de los niños del jardín de infancia Beatriz de Roche en el Institu- to Venezolano de Investigaciones Científicas (IVIC). Act Odont Venez, 43. [Internet]. 2000.

[13] Dacosta O. The prevalence of malocclusion among a population of northern Nigeria school children. West Afr J Med.1999;18(2):91-6.

[14] Onyeaso CO. An epidemiological survey of occlusal anomalies among secondary school children in Ibadan, Nigeria. Odontostomatol Trop. 2003;26(102):25-9.

[15] Colombia. Ministerio de salud. Iv Estudio Nacional de Salud Bucal (eNSAB IV). Situación en Salud Bucal. Tomo I. Bogotá: Ministerio de Salud; 2014.

[16] Bermúdez P. et al. Perfil epidemiológico de la oclusión dental en escolares de 6 a 12 años del Colegio Universidad Cooperativa de Colombia, corregimiento de San Antonio de Prado, 2010. Rev Colomb Investig Odontol. 2011;2(5). Disponible en http://www.rcio.org/index.php/rcio/article/view/ 59/141\%3A\%3Ahtml.

[17] Santiso.Cepero A, Torres-Ulloa M, Álvarez-Curbelo M, Cubero-González R, López-Martín D. Factores de mayor riesgo para maloclusiones dentarias desde la dentición temporal. Revisión bibliográfica. Mediciego. 2010;16(Supl.1): 1-11. Disponible en http://bvs.sld.cu/revistas/mciego/vol16_supl1_10/ pdf/t17.pdf

[18] Baume L. Physiological tooth migration and its significance for the development of the dentition IV. The biogenesis of the overbite. J. Dent. Res. 1050; 440-447.

[19] Anderson GM. Practical Orthodontics. 7th Edition. Ed. Mosby, UsA. 1948; 95-120.

[20] Angle EH. Classification of the malocclusion. Dental Cosmos. 1899; 41 (3): 248-264. Disponible en http://quod.lib.umich.edu/d/dencos/acf8385.0041. 001/266:56? rgn=main; view=image

[21] Medina C. Prevalencia de maloclusiones dentales en un grupo de pacientes pediátricos. Act Odontol Venez. 2010;48(1): 1-19. Disponible en http://www.actaodontologica.com/ediciones/2010/1/pdf/art9.pdf

[22] Aliaga del Castillo A, Mattos MA. Maloclusiones en niños y adolescentes de caseríos y comunidades nativas de la Amazonía de Ucayali, Perú. Rev Per Med Exper Sal Públ. 2011;28(1):87-91.

[23] Botero PM, Vélez N, Cuesta DP, Gómez E, González PA, Cossio, $\mathrm{M}$ et al. Perfil epidemiológico de oclusión dental en niños que consultan a la Universidad Cooperativa de Colombia. Rev Colomb Investig Odontol. 2011;23(1):111-25.

[24] Urrego PA, Jiménez LP. Perfil epidemiológico de la oclusión dental en escolares de Envigado, Colombia. Rev Sal Públ. 2011;13(6):1010-21. 